\title{
A review of palliative radiotherapy for lung cancer and lung metastases
}

\author{
Alexander V. Louie • George Rodrigues • \\ Patrick Cheung • David A. Palma • Benjamin Movsas
}

Received: 11 May 2012 / Accepted: 16 May 2012 / Published online: 28 May 2012

(C) Springer-Verlag 2012

\begin{abstract}
Introduction Treatment of thoracic symptoms related to lung cancer with palliative radiotherapy is routinely performed in conjunction with radiation oncology practice. Various clinical trials, meta-analyses, practice guidelines, consensus statements, and Cochrane reviews have been conducted on different questions related to palliative thoracic radiotherapy considerations including: patient selection, external-beam radiotherapy dose fractionation, endobronchial brachytherapy, and chemotherapy.

Discussion Novel radiotherapy technologies such as stereotactic ablative radiotherapy may allow new populations to benefit from radiotherapy. These new populations include: early stage lung cancer patients not fit for surgery that previously would be treated with best supportive care or palliative approaches and the treatment of oligometastatic lung metastases.
\end{abstract}

\footnotetext{
A. V. Louie $\cdot$ G. Rodrigues $\cdot$ D. A. Palma

Department of Radiation Oncology, London Regional Cancer Program, Western University,

London, ON, Canada

P. Cheung

Department of Radiation Oncology, Odette Cancer Centre, University of Toronto,

Toronto, ON, Canada

B. Movsas

Department of Radiation Oncology, Henry Ford Health System, Detroit, MI, USA

G. Rodrigues $(\bowtie)$

London Health Sciences Centre,

A3-808 790 Commissioners Road East,

London, ON, Canada

e-mail: george.rodrigues@1hsc.on.ca
}

Conclusion The goal of this narrative review is: (1) to review the foundations of what has already been established in palliative thoracic radiotherapy, with a focus on published clinical trials, (2) to review and discuss emerging data and trends in radiotherapeutic options for patients with advanced thoracic malignancies, with a focus on both clinical trials and other forms comparative-effectiveness research, and (3) to discuss active areas of research and future directions for research in this challenging patient population.

Keywords Palliative radiotherapy $\cdot$ Lung cancer . Brachytherapy $\cdot$ Stereotactic ablative radiotherapy . Chemotherapy

\section{Introduction}

Lung cancer is the leading cause of cancer-related death worldwide [1]. Unfortunately, up to $40 \%$ of lung cancer patients present with metastatic disease. While chemotherapy is often considered for these patients, palliative radiotherapy is also important for patients who have not responded to systemic treatment, have relapsed, or have contraindications to potentially toxic drugs. For these radiotherapy patients, the goal of treatment is often to minimize symptom burden from metastatic disease. Similarly, the use of palliative radiotherapy may also be directed to alleviate or prevent symptoms related to local/regional tumor burden on anatomic structures within or near the thorax. Respiratory symptoms such as dyspnea, cough, and airway obstruction; vascular issues such as superior vena cava obstruction; gastrointestinal manifestations such as esophageal obstruction and dysphagia; and pain from chest wall/rib invasion and brachial plexopathy could all potentially benefit from palliative radiation. In total, it is felt that approximately one 
third of patients will experience a global improvement in quality of life $(\mathrm{QoL})$ from palliative lung radiotherapy [2].

Prospective clinical trials assessing the effect of various medical interventions to assess a variety of patient-reported symptom and survival outcomes in advanced thoracic malignancies have been conducted. These interventions can be classified as surgical (i.e., stenting and pleurodesis), radiotherapeutic (external-beam, stereotactic, and brachytherapy), and medical (palliative/best supportive care and chemotherapy) strategies administered either as a standalone or integrated fashion to optimize patient care. Within the context of palliative thoracic radiotherapy for lung cancer, prospective clinical trials have evaluated optimal dose fractionation, the use of endobronchial therapy, the addition of chemotherapy as a radiosensitizing agent during fractionated radiotherapy, and the use of stereotactic ablative radiotherapy (SABR) for oligometastatic disease. Other areas of more limited but emerging comparative-effectiveness research include: patient selection, timing of intervention, evaluation of patient preference, and economic considerations in the selection of treatment modality. In addition, the establishment of advanced radiotherapeutic techniques such as SABR, have afforded radical treatment for lung cancer patients who would otherwise not be treated (or treated with palliative intent) based on other medical co-morbidities. The increasing use and familiarity of these advanced radiation technologies have also opened up the possibility of treatment of oligometastatic lung metastases in a more aggressive fashion.

The primary focus of this review will be on palliative thoracic radiotherapy for non-small cell lung cancer (NSCLC), but will also discuss unique aspects related to recent developments in small cell lung cancer, the oligometastatic disease state, and SABR. This paper can be divided in three broad themes:

1. To review the foundations of what has already been established in palliative thoracic radiotherapy, with a focus on published clinical trials

2. To review and discuss emerging data and trends in radiotherapeutic options for patients with advanced thoracic malignancies, with a focus on both clinical trials and other forms comparative-effectiveness research

3. To discuss active areas of research and future directions for research in this challenging patient population

\section{The foundations of palliative lung radiotherapy}

External-beam radiotherapy

Palliative thoracic radiotherapy is a cornerstone of Radiation Oncology practice, with older estimates from the UK perspective suggesting that $20-25 \%$ of a typical Radiation Oncologist's practice deals with lung-related issues, and $90 \%$ of these treatments are palliative in nature [3]. Initially, fractionation schemes for palliative thoracic radiotherapy in stage IV NSCLC were largely developed empirically, and were subjected to more rigorous clinical trial evaluation in the 1980s and 1990s. Common dose fractionation schedules employed include: $16-17$ Gy in two fractions, 20 Gy in five fractions, $30 \mathrm{~Gy}$ in ten fractions, and 39-45 Gy in 12-15 fractions. Since then, a Cochrane Review [4] was drafted, and then updated [5] to determine the most effective and least toxic palliative radiotherapy regime to improve or prevent thoracic symptoms and/or overall survival. In this review, of 14 randomized control trials [6-19], the authors concluded that there was no strong evidence that any of the reviewed dose fractionation schedules provided greater palliation than the other. Patients with good performance status and treated with higher doses of radiotherapy had a modest increase in survival of $5 \%$ at 1 year and $3 \%$ at 2 years, but at the cost of increased acute toxicity, in particular esophagitis.

Fairchild et al. [20] performed a meta-analysis of 3,473 patients from 13 randomized trials [6-13, 15-19] since 1985 that evaluated different dose fractionation schemes for palliative lung radiotherapy. The authors found that there was a greater likelihood of symptom improvement and a modest benefit in overall survival with radiotherapy schedules of $35 \mathrm{~Gy}_{10} \mathrm{BED}$ (i.e., $30 \mathrm{~Gy}$ in ten fractions) or greater. These findings informed various consensus papers including the American Society for Radiation Oncology (ASTRO) practice guideline [21], and an international consensus statement [22]. The general recommendations from the above reviews and guidelines are that radiotherapy schedules of $35 \mathrm{~Gy}_{10}$ BED (i.e., 30 Gy in ten fractions) or greater should be considered for patients with good performance status. Treatment with a small fraction number is indicated and appropriate in patients who have poor performance status, guarded life expectancy, limited transportation access to treatment, or require rapid treatment completion prior to or in between chemotherapy cycles.

\section{Endobronchial brachytherapy}

Endobronchial brachytherapy (EBB) refers to the placement of a radioactive source within or close to a tumor to provide local high-dose radiotherapy. The treatment involves the placement of a catheter within the lumen of the airway to facilitate the placement of a high-dose rate radioactive source in anatomical proximity to the area of concern. EBB employs the principle of the inverse square law, where there is a rapid dose fall-off from the radioactive source, and as such, there is limited dose deposited in the remainder of the lung. This treatment option is a particularly attractive 
one for the palliation of endobronchial lesions that are causing dyspnea, hemoptysis, cough, or obstructive pneumonitis. However, there are several prerequisites to this treatment: (1) the ability to pass the endobronchial tube through the length of the obstructive lesion, (2) the skills and training necessary to perform the procedure, (3) adequate facilities/access to OR time, and (4) trained support staff to assist in the procedure.

Recent reviews of randomized control trials comparing EBB with external beam radiotherapy as upfront palliative measures in advanced lung cancer did not find any benefit of EBB when used as a primary modality or as an adjunct to external beam radiotherapy $[23,24]$. These reviews and other guidelines support the use of EBB when there is endobronchial obstruction, given the improved reexpansion rates in a randomized clinical trial [25], as well as in the context of when a patient has failed external beam radiotherapy and has recurrent endobronchial obstruction. While EBB is a reasonable treatment option in these scenarios, the recently published ASTRO guideline reiterates that there is no consensus on the ideal prescription regimen, and that $\mathrm{EBB}$ is inferior to external beam radiotherapy in the initial palliation of thoracic symptoms [21].

\section{Chemotherapy}

Chemotherapy has been established as a standard of care for patients with metastatic NSCLC. In multiple randomized clinical trials comparing platinum-based chemotherapy with best supportive care (BSC), a survival benefit is noted for younger patients with good performance status without compromising QoL [26-28]. This benefit appears to be maintained even in the elderly with a moderate performance status [29]. The advantage of palliative chemotherapy for stage IV NSCLC patients is not limited to a benefit in survival and QoL. In the European Big Lung Trial, where 725 patients with advanced NSCLC were assigned to BSC or cisplatin-based chemotherapy, fewer patients receiving systemic treatment required palliative radiotherapy (47\%) when compared to the BSC group (74\%) [26].

A review of the literature reveals only one phase III randomized clinical trial assessing the use of concurrent chemotherapy and palliative radiotherapy for NSCLC [30]. In this study, patients were treated with 20 Gy in five fractions with or without continuous infusion 5fluorouracil. Not only was there no difference in palliation of symptoms and survival outcomes, the concurrent chemoradiotherapy arm were associated with a significant increase in treatment-related toxicity. In routine practice, stage IV NSCLC patients are often treated in a sequential fashion with both palliative radiotherapy and systemic chemotherapy. In general, symptomatic patients unsuitable for radical treatment receive upfront palliative radiotherapy, while chemotherapy is given upfront for patients who are either asymptomatic or have minimal symptoms. Additionally, palliative radiotherapy can be "sandwiched" between chemotherapy cycles for the palliation of symptoms that occur during a course of chemotherapy.

Small cell lung cancer

SCLC represents approximately $15-20 \%$ of primary lung malignancies, and is characterized by its high sensitivity to chemotherapy, rapid rate of growth, and tendency to metastasize early. In the situation where there is a contraindication to chemotherapy, SCLC may be palliated with radiation, as SCLC is also known to be quite radiosensitive. This is, however, with the recognition that survival outcomes with radiotherapy alone are dismal [31]. In the setting of palliative thoracic radiotherapy for extensive stage disease, common dose fractionation schedules used for the palliation of non-small cell lung cancer (see above) can be utilized for small cell cancer.

The use of prophylactic cranial irradiation following concurrent chemoradiotherapy to the thorax has been well established in SCLC patients with limited stage disease, with a meta-analysis showing a survival benefit of $5 \%$ at 3 years [32]. A similar benefit has been recently established for extensive stage SCLC patients with a partial response to initial chemotherapy, reducing the risk of symptomatic brain metastases, and significantly improving both disease-free and overall survival [33].

\section{Emerging trends in palliative lung radiotherapy}

\section{Patient preferences}

As modern Medicine moves away from the traditionally patriarchal approach of "the doctor knows best", patients are more inclined to be active participants in their own health and medical decision-making - a concept known as shared decision-making. Tang et al. reported the evaluation of a decision aid for patients with metastatic lung cancer on radiotherapy treatment fraction length. The tool enumerated the pros and cons of two palliative thoracic radiotherapy fractionation schedules (17 Gy in two fractions vs. 39 Gy in 13 fractions), with regards to various patient, technical, and cost endpoints [34]. The patients participating in the study were split in their decision, with $55 \%$ choosing the longer fractionation schedule citing improved local control and survival. Conversely, $45 \%$ selected the shorter fractionation schedule because of improved convenience, lower cost, and better symptom control. Interestingly, more than half of the patients ultimately were treated with the alternate treatment option at the advice of the treating physician. This study 
highlights that today's patient can often be highly informed (or sometimes misinformed) by the plethora of information publically available, and thus it is important for physicians to educate and inform them of their options. They conclude that the most sound treatment decision is one in which both the physician and patient are in agreement of the proposed plan of action.

\section{Economic considerations}

Given the ever-rising costs in health care, it is important for doctors to be gatekeepers in resource allocation, weighing the factors of patient benefit and economic considerations. Two important economic analyses have been published on the topic of palliative thoracic radiation. Recently, a cost-utility analysis was performed based on clinical trial results comparing $16 \mathrm{~Gy}$ in two fractions versus $30 \mathrm{~Gy}$ in ten fractions [35]. In this type of analysis, survival and utility (QoL) are evaluated and compared to the cost of the treatment to determine an incremental cost-utility ratio expressed in dollars per quality adjusted life years (QALY). The authors concluded that while the use of the short radiotherapy scheme was appropriate in many situations, patients with a good performance status should be considered for the longer schedule, given that it was within the $\$ 50,000 /$ QALY threshold that many would consider justifiable on economic grounds.

Similarly, Coy et al. performed a cost-utility analysis of high-dose palliative radiotherapy versus best supportive care in advanced NSCLC [36]. This study is important in that it provided estimates of cost-effectiveness and cost-utility ratios in a framework that cannot be performed in the context of a randomized control trial, as many would consider withholding palliative radiotherapy in a randomized manner unethical. The study found that from both the societal and the clinic perspectives, palliative thoracic radiotherapy was also within the $\$ 50,000 /$ QALY threshold.

\section{Stereotactic ablative radiotherapy}

The outcomes for patients with stage I NSCLC who are left untreated are dismal, with a population-based study demonstrating a 5 -year overall survival of only $6 \%$ [37]. Treatment with conventionally fractionated external beam radiotherapy demonstrates only marginal improvements in outcome when compared to no treatment [38]. Elderly and comorbid patients with lung cancer are less likely to receive radiotherapy due to concerns about comorbidity, frailty, or the perceived efficacy of treatment with palliative treatment deferred until the development of symptoms [39]. The emergence of novel technology in radiation oncology has afforded new treatment options for such patients. SABR (also commonly referred to as stereotactic body radiotherapy, or SBRT) exploits advances in technology to deliver multiple beams of high-dose, highly conformal radiation usually in five fractions or less. SABR has been shown to be safe and an excellent treatment option for the elderly [40], patients with severe COPD [41], or previous pneumonectomy [42]. In fact, a Markov model argues that withholding (i.e., best supportive care approach) SABR from stage I NSCLC patients based on advanced age and severe COPD is not justified, given the potential for a benefit in survival and health-related quality-of-life with SABR treatment [43].

Because of how effective SABR is for patients with stage I NSCLC, there has been increasing interest in exploring its use in patients with oligometastatic disease. This disease state was first defined in 1995 and refers to a stage of disease where cancer has spread beyond the site of origin, but is not yet widely metastatic [44]. With a limited burden of metastatic disease, radical treatment of these sites either surgically (metastatectomy) or with SABR could result in long-term disease control, and even cure for a limited subset of patients [45]. For instance, a recent report described excellent outcomes for elect stage IV NSCLC patients with a solitary brain metastasis that are treated with resection and whole brain radiotherapy, and aggressive measures in the thorax [46]. Similarly, in a recent phase I/II patients treated with SABR for one to three lung metastases from a variety of primary tumors, local control was remarkably $96 \%$ at 2 years, and 2-year survival was $39 \%$ [47]. The results of these studies must be taken within the context, however, that they represent a highly selected patient population.

To further elucidate the role of SABR in oligometastatic patients, a multi-institutional clinical trial, Stereotactic Ablative Radiotherapy for Comprehensive Treatment of Oligometastatic Tumors (SABR-COMET) is now open for accrual [48]. In this randomized phase II study, patients with up to five metastases (no more than three within one organ system) are randomized to either the standard of care (palliative radiotherapy to any symptomatic site, with further chemotherapy at the discretion of the Medical Oncologist) versus SABR to all known metastatic deposits with further chemotherapy at the discretion of the Medical Oncologist. In this trial of 99 patients, the primary endpoint is overall survival, with secondary endpoints evaluating QoL, toxicity, number of cycles of chemotherapy required, and progression-free survival.

\section{Future directions}

Timing and/or combination of treatments

Given recent advances in oral targeted systemic agents, we are likely to see data emerging on the concurrent or sequential use of these agents along with palliative external beam radiotherapy for advanced lung cancer patients. In general, these medications appear to be well tolerated, and are likely 
to play a large role in the era of personalized medicine. Similarly, in the context of oligometastatic disease, there is a paucity of data concerning if and when chemotherapy should be combined with SABR to optimize patient outcomes. The role of chemotherapy in this context would be to eradicate microscopic disease (rather than to radiosensitize) that ablative treatments would not otherwise encompass.

The role of advanced radiotherapy technologies

The paradigm in palliative thoracic radiotherapy is efficient treatment planning in which target volumes are defined via easily accessible and convenient radiographic techniques such as chest X-ray, bronchoscopy, and/or computed tomography, with the addition of a wide margin. The benefit of the use of advanced technologies such as intensity-modulated radiotherapy, image-guided radiotherapy, four-dimensional computed tomography, and positron emission tomography. However, given the amount of time and/or complexity required to employ these modalities, any therapeutic benefit from a radiotherapy point of view may be outweighed by delays in treatment. Limited information is available regarding the implementation of such technologies in the routine care of patients requiring palliative radiotherapy.

\section{Conclusions}

Radiotherapy appears to be an effective and efficient means to palliate symptoms caused by local/regional spread of lung cancer. Short fractionation schemes are indicated for symptomatic patients with poor performance status, with longer higher dose regimes reserved for those who are fit. Advances in radiation technology have enabled the radical treatment of patients who were once considered palliative either because of their disease burden, or because of co-morbid illness. While clinical trials mature, the emergence of alternate forms of research such as comparative-effectiveness, cost-utility analyses, and patient preference take on greater importance to guide clinicians and their patients to the most appropriate decisions for individual scenarios.

Acknowledgments The authors acknowledge Abhinay Sathya for his assistance in the preparation of the manuscript.

Conflict of interest statement Dr. Movsas's institution has a research agreement with Varian Inc. No other conflicts of interest related to this work exist.

\section{References}

1. Jemal A, Bray F, Center MM, Ferlay J, Ward E, Forman D (2011) Global cancer statistics. CA Cancer J Clin 61(2):69-90
2. Langendijk JA, ten Velde GP, Aaronson NK et al (2000) Quality of life after palliative radiotherapy in non-small cell lung cancer: a prospective study. Int J Radiat Oncol Biol Phys 47:149-155

3. Maher EJ, Timothy A, Squire CJ, Goodman A, Karp SJ, Paine CH, Ryall R, Read G (1993) Audit: the use of radiotherapy for NSCLC in the UK. Clin Oncol 5(2):72-79

4. Macbeth F, Toy E, Coles B, Melville A, Eastwood A (2006) Palliative radiotherapy regimens for non-small cell lung cancer. Cochrane Database Syst Rev. doi:10.1002/14651858.CD002143

5. Lester JF, Macbeth F, Toy E, Coles B (2009) Palliative radiotherapy regimens for non-small cell lung cancer. Cochrane Database Syst Rev. doi:10.1002/14651858.CD002143.pub2

6. Simpson JR, Francis ME, Perez-Tamayo R, Marks RD, Rao DV (1985) Palliative radiotherapy for inoperable carcinoma of the lung: final report of a RTOG multi-institutional trail. Int J Radiat Oncol Biol Phys 11:751-758

7. Teo P, Tai TH, Choy D, Tsui KH (1988) A randomized study on palliative radiation therapy for inoperable non small cell carcinoma of the lung. Int J Radiat Oncol Biol Phys 14:867-871

8. Abratt RP, Shepherd LJ, Mameena Salton DG (1995) Palliative radiation for stage 3 non-small cell lung cancer-a prospective study of two moderately high dose regimens. Lung Cancer 13 (2):137-143

9. Medical Research Council Lung Cancer Working Party (1991) Inoperable non-small-cell lung cancer (NSCLC): a medical research council randomised trial of palliative radiotherapy with two fractions or ten fractions. Br J Cancer 63:265-270

10. Medical Research Council Lung Cancer Working Party (1992) A Medical Research Council (MRC) randomised trial of palliative radiotherapy with two fractions or a single fraction in patients with inoperable non-small-cell lung cancer (NSCLC) and poor performance status. Br J Cancer 65:934-9541

11. Medical Research Council Lung Cancer Working Party (1996) Randomized trial of palliative two-fraction versus more intensive thirteen fraction radiotherapy for patients with inoperable nonsmall cell lung cancer and good performance status. Clin Oncol 8:167-175

12. Nestle U, Nieder N, Walter K, Abel U, Ukena D, Sybrecht GW, Schnabel K (2000) A palliative accelerated irradiation regimen for advanced non-small cell lung cancer vs. conventionally fractionated 60 Gy: results of a randomized equivalence study. Int J Radiat Oncol Biol Phys 48(1):95-103

13. Rees GJ, Devrell CE, Barley VL, Newman HFV (1997) Palliative radiotherapy for lung cancer; two versus five fractions. Clin Oncol 9:90-95

14. Reinfuss M, Glinski B, Kowalska T, Kulpa J, Zawila K, Reinfuss K, Dymek P, Herman K, Skolyszewski J (1999) Radiotherapy in stage III, unresectable, asymptomatic non-small cell lung cancer. Final results of a prospective randomized study of 240 patients. [Radiotherapie du cancer bronchique non a petites cellules de stade III, inoperable, asymptomatique. Resultats definitifs d'un essai prospectif randomise (240 patients). Cancer Radiotherapie 3:475479

15. Bezjak A, Dixon P, Brundage M, Tu DS, Palmer MJ, Blood P, Grafton C, Lochrin C, Leong C, Mulroy L, Smith C, Wright J, Pater JL (2002) Randomized phase III trial of single versus fractionated thoracic radiation in the palliation of patients with lung cancer (NCIC CTGSC.15). Int J Radiat Oncol Biol Phys 54:719728

16. Sundstrom S, Bremnes R, Aasebo U, Aamdal S, Hatlevoll R, Brunsvig P, Johannessen DC, Klepp O, Fayers PM, Kaasa S (2004) Hypofractionated palliative radiotherapy (17 Gy per 2 fractions) in advanced non-small cell lung carcinoma is comparable to standard fractionation for symptom control and survival: a national phase III trial. J Clin Oncol 22:801-810 
17. Erridge SC, Gaze MN, Price A, Kelly CG, Kerr GR, Cull A, MacDougall RH, Howard GC, Cowie VJ, Gregor A (2005) Symptom control and quality of life in people with lung cancer: a randomised trial of two palliative radiotherapy fractionation schedules. Clin Oncol 17:61-67

18. Kramer GW, Wanders SL, Noordijk EM, Vonk EJ, van Houwelingen HC, van den Hout WB, Geskus RB, Scholten M, Leer JW (2005) Results of the Dutch National study of the palliative effect of irradiation using two different treatment schemes for non-small cell lung cancer. J Clin Oncol 23:2962-2970

19. Senkus-Konefka E, Dziadziuszko R, Bednaruk-Mlynski E, Pliszka A, Kubrak J, Lewandowska A, Malachowski K, Wierzchowski M, Matecka-Nowak M, Jassem J (2005) A prospective randomised study to compare two palliative radiotherapy schedules for nonsmall cell lung cancer (NSCLC). Br J Cancer 92:1038-1045

20. Fairchild A, Harris K, Barnes E, Wong R, Lutz S, Bezjak A, Cheung P, Chow E (2008) Palliative thoracic radiotherapy for lung cancer: a systematic review. J Clin Oncol 26(24):4001-4011

21. Rodrigues G, Videtic G, Bradley J et al (2011) Palliative thoracic radiotherapy in lung cancer: an American society for radiation oncology evidence-based clinical practice guideline. Pract Radiat Oncol 1:60-71

22. Rodrigues G, Macbeth F, Burmeister B, Kelly K, Bezjak A, Langer C, Hahn C, Vichare A, Movsas B (2012) International practice survey on palliative lung radiotherapy: third international consensus workshop on palliative radiotherapy and symptom control. Clin Lung Cancer 13(3):225-235

23. Ung Y, Yu E, Falkson C et al (2006) The role of high-dose-rate brachytherapy in the palliation of symptoms in patients with nonsmall-cell lung cancer: a systematic review. Brachytherapy 5 (3):189-202

24. Cardona AF, Reveiz L, Ospina EG, Ospina V, Yepes A (2008) Palliative endobronchial brachytherapy for non-small cell lung cancer. Cochrane Database Sys Rev. doi:10.1002/14651858.CD004284pub2

25. Rodrigues G, Movsas B (2012) Future directions in palliative thoracic radiotherapy. Curr Opinion Support Palliative Care 6 (1):91-96

26. Spiro S, Rudd R, Souhami R et al (2004) Chemotherapy versus supportive care in advanced non-small cell lung cancer: improved survival without detriment to quality of life. Thorax 59(10):828836

27. Group Non-small Cell Lung Cancer Collaborative (1995) Chemotherapy in non-small cell lung cancer: a meta-analysis using updated data on individual patients from 52 randomised clinical trials. BMJ 311(7010):899

28. Shanafelt TD, Loprinzi C, Marks R, Novotny P, Sloan J (2004) Are chemotherapy response rates related to treatment-induced survival prolongations in patients with advanced cancer? J Clin Oncol 22 (10):1966-1974

29. The Elderly Lung Cancer Vinorelbine Italian Study Group (1999) Effects of vinorelbine on quality of life and survival of elderly patients with advanced non-small-cell lung cancer. J Natl Cancer Inst 91(1):66-72

30. Ball D, Smith J, Bishop J, Olver I, Davis S, O’Brien P, Bernshaw D, Ryan G, Millward M (1997) A phase III study of radiotherapy with and without continuous-infusion fluorouracil as palliation for non-small-cell lung cancer. Br J Cancer 75(5):690-697

31. Kepka L, Sprawka A, Casas F, Abdel-Wahab S, Agarwal JP, Jeremic B (2009) Radiochemotherapy in small-cell lung cancer. Expert Rev Anticancer Ther 9(10):1379-1387

32. Auperin A, Arriagada R, Pignon JP, Le Pechoux C, Gregor A, Stephans RJ, Kristjansen PEG, Johnson BE, Ueoka H, Wagner H, Aisner J (1999) Prophylactic cranial irradiation for patients with small-cell lung cancer in complete remission. N Eng J Med 341 (7):476-484

33. Slotman B, Faivre-Finn C, Kramer G, Rankin E, Snee M, Hatton M, Postmus P, Collette L, Musat E, Senan S, EORTC Radiation Oncology Group and Lung Cancer Group (2007) Prophylactic cranial irradiation in extensive stage small cell lung cancer patients. N Engl J Med 357(7):664-672

34. Tang JI, Shakespeare TP, Lu JJ, Chan YH, Lee KM, Wong LC, Mukherjee RK, Back MF (2008) Patients' preference for radiotherapy fractionation schedule in the palliation of symptomatic unresectable lung cancer. J Med Imaging Radiat Oncol 52(5):497-502

35. Coy P, Schaafsma J, Schofield JA (2000) The cost-effectiveness and cost-utility of high-dose palliative radiotherapy for advanced non-small-cell lung cancer. Int J Radiat Oncol Biol Phys 48 (4):1025-1033

36. van den Hout WB, Kramer GW, Noordijk EM, Leer JW (2006) Cost-utility analysis of short- versus long-course palliative radiotherapy in patients with non-small cell lung cancer. J Natl Cancer Inst 98:1786-1794

37. Raz DJ, Zell JA, Ou SH, Gandara DR, Anton-Culver H, Jablons DM (2007) Natural history of stage I non-small cell lung cancer: implications for early detection. Chest 132(1):193-199

38. Rowell NP, Williams C (2001) Radical radiotherapy for stage I/II non-small cell lung cancer in patients not sufficiently fit for or declining surgery (medically inoperable). Cochrane Database Syst Rev. doi:10.1002/14651858.CD002935

39. Owonikoko TK, Ragin CC, Belani CP, Oton AB, Gooding WE, Taioli E, Ramalingam SS (2007) Lung cancer in elderly patients: an analysis of the surveillance, epidemiology, and end results database. J Clin Oncol 25(35):5570-5577

40. Haasbeek CJ, Lagerwaard FJ, Antonisse ME, Slotman BJ, Senan S (2010) Stage I nonsmall cell lung cancer in patients aged $>$ or $=75$ years: outcomes after stereotactic radiotherapy. Cancer 116 (2):406-414

41. Palma D, Lagerwaard F, Rodrigues G, Haasbeek C, Senan S (2012) Curative treatments of stage I non-small-cell lung cancer in patients with severe COPD: stereotactic radiotherapy outcomes and systematic review. Int J Radiat Oncol Biol Phys 82(3):11491156

42. Haasbeek CJ, Lagerwaard FJ, de Jaeger K, Slotman BJ, Senan S (2009) Outcomes of stereotactic radiotherapy for a new clinical stage I lung cancer arising postpneumonectomy. Cancer 115:587594

43. Louie AV, Rodrigues G, Hannouf M, Lagerwaard F, Palma D, Zaric GS, Haasbeek C, Senan S (2011) Withholding stereotactic radiotherapy in elderly patients with stage I non-small cell lung cancer and co-existing COPD is not justified: outcomes of a Markov model analysis. Radiother Oncol 99(2):161-165

44. Hellman S, Weichselbaum (1995) Oligometastases. J Clin Oncol 13(1):8-10

45. Macdermed DM, Weichselbaum RR, Salama JK (2008) A rationale for the targeted treatment of oligometastases with radiotherapy. J Surg Oncol 98(3):202-206

46. Louie AV, Rodrigues G, Yaremko B et al (2009) Management and prognosis in synchronous solitary resected brain metastasis from non-small-cell lung cancer. Clin Lung Cancer 10(3):174-179

47. Rusthoven KE, Kavanagh BD, Burri SH et al (2009) Multiinstitutional phase I/II trial of stereotactic body radiation therapy for lung metastases. J Clin Oncol 27(10):1579-1584

48. Palma D (2011) Stereotactic ablative radiotherapy for comprehensive treatment of oligometastatic tumors (SABR-COMET). Clinicaltrials.gov. clinicaltrials.gov/ct2/show/NCT01446744. Accessed 4 May 2012 